\title{
Expression of hyperpolarization-activated cyclic nucleotide-gated channel isoforms in a canine model of atrial fibrillation
}

\author{
WEI HE, JIAN ZHANG, TIANYI GAN, GUOJUN XU and BAOPENG TANG \\ Pacing Electrophysiology Division, First Affiliated Hospital of Xinjiang Medical University, \\ Ürümqi, Xinjiang 830054, P.R. China
}

Received May 21, 2014; Accepted December 17, 2014

DOI: $10.3892 /$ etm.2016.3290

\begin{abstract}
The aim of the present study was to analyze the mRNA and protein expression levels of atrial hyperpolarization-activated cyclic nucleotide-gated (HCN) channel isoforms in the left atrial muscle of dogs with multiple organ failure. A total of 14 beagle dogs with multiple organ failure, including seven cases with sinus rhythm and seven cases with atrial fibrillation (AF), underwent surgery to remove a sample of left atrial appendage tissue. The expression levels of a number of HCN channel subtypes were subsequently measured using quantitative polymerase chain reaction and western blot analysis. The mRNA and protein expression levels of HCN2 and HCN4 increased significantly in the AF group when compared with the sinus rhythm group. However, expression of the HCN1 isoform was not detected. Therefore, increased expression levels of HCN2 and HCN4 may be important molecular mechanisms in the pathogenesis of AF, which were associated with differences in patients with valvular heart disease.
\end{abstract}

\section{Introduction}

Atrial fibrillation (AF) is the most common type of sustained cardiac arrhythmia, affecting 1-2\% of the general population (1). The prevalence of AF increases with age $(2,3)$ and the condition is a known risk factor for a number of cardiovascular diseases, including a five-fold risk of stroke, three-fold incidence of congestive heart failure and an increased rate of mortality (4).

Previous studies have found that the occurrence of AF affects atrial activity, causing the atria to quiver persistently or relapse into fibrillation soon after. Wijffels et al (1) first described this process as atrial electrical remodeling,

Correspondence to: Dr Baopeng Tang, Pacing Electrophysiology Division, First Affiliated Hospital of Xinjiang Medical University, 137 Southern Liyu Shan Road, Ürümqi, Xinjiang 830054, P.R. China E-mail: tangbpxj@163.com

Key words: multiple organ failure, atrial fibrillation, hyperpolarization-activated cyclic nucleotide-gated channel, atrial electrical remodeling observing that AF was able to induce electrophysiological changes itself, which in turn promoted further AF development and maintenance ('AF begets AF') (5). The theory of atrial electrical remodeling has become increasingly accepted and this self-perpetuating process is considered to be an important mechanism of AF maintenance.

Changes to a number of ion channel currents may result in electrical remodeling, including the L-type calcium current, the transient outward potassium current when downregulated and the inward rectifier potassium current when upregulated (6). Previous studies have found that the hyperpolarization current $\left(\mathrm{I}_{\mathrm{f}}\right)$ is associated with atrial electrical remodeling in patients with AF (7-9). The $I_{f}$ current is a depolarizing sodium-potassium mixed non-specific current with hyperpolarization-activated intracellular cAMP regulatory characteristics, which is involved in the depolarization of cardiac pacemaker cells (10). Increased $I_{f}$ current activity may lead to an increase in myocardial tissue self-perpetuation, which commonly results in a tachyarrhythmia. The molecular propagators of the $\mathrm{I}_{\mathrm{f}}$ current are hyperpolarization-activated cyclic nucleotide-gated (HCN) channels (11). Therefore, the inhibition of HCN channels has become a focus of study for the treatment of arrhythmia (6). However, few studies have investigated changes in the expression of HCN channel isoforms in atrial cells following AF. The aim of the present study was to investigate the molecular mechanisms of AF and atrial electrical remodeling by comparing the mRNA and protein expression levels of $\mathrm{HCN}$ channel isoforms (HCN1, 2 and 4) in left atrial tissues from a canine model of AF.

\section{Materials and methods}

Animal model establishment. A total of 14 healthy beagle dogs $(\mathrm{n}=2$ for the preliminary experiment; $\mathrm{n}=12$ for the remaining experiments) weighing $10.1 \pm 2.9 \mathrm{~kg}$ and aged $1.5-2.5$ years were divided into two groups. All the dogs were implanted with pacemakers in a subcutaneous neck pocket using a previously described procedure $(12,13)$. Following a 24-h recovery period, seven dogs were subjected to continuous right atrial pacing at $400 \mathrm{bpm}$ for 50 days (AF group). In the remaining seven $\operatorname{dog}$ s, the pacemaker was not activated and a normal sinus rhythm heart beat was maintained (sham control group). The pacemaker was programmed to stimulate the atrium at $400 \mathrm{bpm}$, (cycle length, $150 \mathrm{msec}$ ) using $0.42 \mathrm{msec}$ square 
wave pulses at twice-threshold current. The surface electrocardiogram was verified after $24 \mathrm{~h}$, and then weekly to ensure continuous 1:1 atrial capture. The dogs were euthanized with an overdose of $\alpha$-chloralose $(350 \mathrm{mg} / \mathrm{kg}$ ). The heart was removed, and the right atrial free wall and sinoatrial node were isolated. The left atrial appendage tissues were separated (weight, $200 \mathrm{mg}$ ), and the samples were rapidly frozen in liquid nitrogen and stored at $-80^{\circ} \mathrm{C}$. All animal experiments were performed in accordance with the protocols approved by the Animal Care Committee of the Institute of Zoology at the Chinese Academy of Sciences (Beijing, China).

RNA isolation. RNA was isolated from 0.1-1.0-g samples of left atrial tissue using TRIzol reagent (Invitrogen Life Technologies, Carlsbad, CA, USA), following which phenol-chloroform extraction and isopropanol precipitation were conducted. Genomic DNA was eliminated through incubation in DNase $\mathrm{I}\left(0.1 \mathrm{U} / \mathrm{ml}, 37^{\circ} \mathrm{C}\right)$ for $30 \mathrm{~min}$, followed by acid-phenol-chloroform extraction. The RNA was quantified by measuring the spectrophotometric absorbency at $260 \mathrm{~nm}$, while the purity was confirmed by $\mathrm{A}_{260} / \mathrm{A}_{280}$ ratio assessment. DNA integrity was evaluated with ethidium bromide staining on a denaturing agarose gel, and the RNA samples were stored at $-80^{\circ} \mathrm{C}$ in RNAsecure Resuspension Solution (Ambion Life Technologies, Carlsbad, CA, USA).

Quantitative polymerase chain reaction (qPCR). A 1- $\mu \mathrm{g}$ sample of reference RNA was reverse transcribed into cDNA using a reverse transcriptase kit (A3500; Promega Corporation, Madison, WI, USA). The design and synthesis details of the primers used are shown in Table I; the primers were synthesized by Takara Bio, Inc. (Otsu, Japan). The cDNA sample was diluted five times and $2 \mu 1$ was added to the qPCR system (25 $\mu \mathrm{l}$ ), which also contained $12.5 \mu \mathrm{l}$ SYBR Green qPCR Master Mix (Ruian BioTechnologies Co., Ltd., Shanghai, China), $0.5 \mu \mathrm{l}$ upstream primer $(10 \mu \mathrm{M}), 0.5 \mu \mathrm{l}$ downstream primer $(10 \mu \mathrm{M})$ and $9.5 \mu \mathrm{ldd} \mathrm{H}_{2} \mathrm{O}$. The reaction was conducted as follows: Denaturation for $2 \mathrm{~min}$ at $95^{\circ} \mathrm{C}$, followed by $35 \mathrm{PCR}$ cycles of denaturation for $10 \mathrm{sec}$ at $95^{\circ} \mathrm{C}$, annealing for $30 \mathrm{sec}$ at $58,56.5,59$ and $57^{\circ} \mathrm{C}$ for $\mathrm{HCN} 1, \mathrm{HCN} 2, \mathrm{HCN} 4$ and $\beta$-actin, respectively, and a final extension for $40 \mathrm{sec}$ at $72^{\circ} \mathrm{C}$. The mRNA expression levels were calculated for each HCN channel isoform using the comparative $\mathrm{Ct}$-method. The length of the target gene products (HCN2, $143 \mathrm{bp}$; HCN4, $132 \mathrm{bp}$; $\beta$-actin, $152 \mathrm{bp}$ ) were consistent with the theoretical values. PCR products were visualized under UV light using ethidium bromide staining in $1.5 \%$ agarose gel. Images were captured with an Olympus DP22 camera (Olympus Corporation, Tokyo, Japan) and the band intensity was determined using Quantity One software (Bio-Rad Laboratories, Inc., Hercules, CA, USA). A DNA MassMarker (100 ng; Takam Co., Japan) was used to determine the size and quantity of the DNA bands and to create standard curves in each experiment for absolute quantification.

Western blot analysis. Total protein was extracted using a western blot kit (bs-0542; Beijing Bioss Science and Technology Co., Ltd., Beijing, China) according to the manufacturer's instructions, and the protein concentration was assessed using the bicinchoninic acid method. SDS-PAGE was used to separate the total protein content of the 50- $\mu \mathrm{g}$ samples in each well. The separated protein samples were transferred by electroporation onto a nitrocellulose membrane. Following elution, 5\% non-fat dry milk and Tris-buffered-saline were used for blocking. The membranes were incubated overnight at $4{ }^{\circ} \mathrm{C}$ with specific rabbit primary antibodies against HCN1 (cat. no. ab19405), HCN2 (cat. no. ab136839), HCN4 (Abcam, Cambridge, UK; cat. no. ab69054), SR-4 antibody (cat. no. sc-376158; Santa Cruz Biotechnology, Inc., Dallas, TX, USA) and $\beta$-actin (Wuhan Boster Biological Technology Ltd., Wuhan, China; cat. no. 10082003) at $1 \mathrm{ml} / \mathrm{mg}$. Subsequently, the membranes were incubated for $1 \mathrm{~h}$ with a horseradish peroxidase-conjugated secondary antibody (goat anti-rabbit IgG; Wuhan Boster Biological Technology, Ltd.; cat. no. BA1055). Finally, the membranes underwent visualization using a 3,3'-diaminobenzidine colorimetric gel imaging system (Bio-Rad Laboratories, Inc.) in order to quantify the hybridization signal.

Statistical analysis. Measurement data are expressed as the mean \pm standard deviation. The t-test was used to compare between groups or non-parametric test, the $\chi^{2}$ test was used in categorical data. Associations between variables were detected using linear correlation analysis. Statistical analysis was performed with SPSS 16.0 statistical software (SPSS, Inc., Chicago, IL, USA), where $\mathrm{P}<0.05$ was considered to indicate a statistically significant difference.

\section{Results}

$m R N A$ expression. PCR products of the $\mathrm{HCN}$ channel isoforms are shown in Table I. HCN1 mRNA expression was not detected in the canine atrial muscle samples, which may be due to very low levels of expression. Therefore, only the mRNA expression levels of HCN2 and HCN4 were compared. The mRNA expression levels of $\mathrm{HCN} 2$ and $\mathrm{HCN} 4$ showed a statistically significant increase in the AF group when compared with the sham control group $(\mathrm{P}<0.05$; Table II).

Using a 100-bp DNA marker as a reference, the lengths of the $\beta$-actin, $\mathrm{HCN} 2$ and $\mathrm{HCN} 4 \mathrm{mRNA}$ fragments were found to be 152, 143 and 132 bp, respectively (Fig. 1).

Protein expression. HCN1 protein expression was not detected in the canine atrial muscle samples. Western blot analyses of the HCN2 and HCN4 proteins are shown in Fig. 2, where $\beta$-actin was used a reference to calculate the $\mathrm{HCN} 2$ and $\mathrm{HCN} 4$ protein expression levels. The protein expression levels of $\mathrm{HCN} 2$ and $\mathrm{HCN} 4$ showed a statistically significant increase in the AF group when compared with dogs in the sham control group $(\mathrm{P}<0.05)$.

\section{Discussion}

HCN channels are the molecular propagators of the $I_{f}$ current (6). There are four $\mathrm{HCN}$ channel isoforms in mammals, HCN1-4. Configurations of HCN channels in different species and tissues vary considerably. The majority of $\mathrm{HCN} 3$ channels are located in central nervous system tissues, while HCN1, $\mathrm{HCN} 2$ and HCN4 are expressed abundantly in the heart tissue. The present study revealed that HCN1 expression was 
Table I. Primer sequences used for qPCR.

\begin{tabular}{|c|c|c|c|}
\hline Gene & $\begin{array}{c}\text { Primer } \\
\text { sequence }\end{array}$ & $\begin{array}{c}\text { Product } \\
\text { length (bp) }\end{array}$ & $\begin{array}{c}\text { Annealing } \\
\text { temperature }\left({ }^{\circ} \mathrm{C}\right)\end{array}$ \\
\hline \multirow[t]{2}{*}{$\beta$-actin } & F: 5' AAGGACCTGTATGCCAACACA 3' & & \\
\hline & R: 5' ATCCACACAGAATACTTGCGTT 3' & 152 & 57 \\
\hline \multirow[t]{2}{*}{ HCN1 } & F: 5' GACGCTATGGGCTATGAGTTAC 3' & & \\
\hline & R: 5' AGTCCAGGTAGCCCTTTAGGT 3' & 199 & 58 \\
\hline \multirow[t]{2}{*}{$\mathrm{HCN} 2$} & F: 5' ACTGCTGGGTCTCCATCAAC 3' & & \\
\hline & R: 5' CGTCAGCCAGATGTCCGT 3' & 143 & 56.5 \\
\hline \multirow[t]{2}{*}{ HCN4 } & F: 5' CATCCAGTCCCTGGACTCGT 3' & & \\
\hline & R: 5' TGGTAGCGGTGCTCGTAGTAG 3' & 132 & 59 \\
\hline
\end{tabular}

qPCR, quantitative polymerase chain reaction; $\mathrm{HCN}$, hyperpolarization-activated cyclic nucleotide-gated; F, forward; R, reverse.

Table II. Comparison of mRNA expression levels of $\mathrm{HCN}$ isoforms in the AF and SC groups.

\begin{tabular}{lccc}
\hline Category & Dogs $(\mathrm{n})$ & $\mathrm{HCN} 2$ & $\mathrm{HCN} 4$ \\
$\mathrm{~s}$ SC & 5 & $1.27 \pm 0.11$ & $1.14 \pm 0.14$ \\
$\mathrm{AF}$ & 7 & $2.23 \pm 0.22^{\mathrm{a}}$ & $2.97 \pm 0.21^{\mathrm{a}}$ \\
P-value & - & 0.03 & 0.01 \\
\hline
\end{tabular}

Results are expressed as the mean \pm standard deviation. ${ }^{\mathrm{a}} \mathrm{P}<0.05$, vs. $\mathrm{SC}$ group. HCN, hyperpolarization-activated cyclic nucleotide-gated; $\mathrm{SC}$, sham control group; $\mathrm{AF}$, atrial fibrillation.

not detectable in the atrial muscle of dogs in the AF or sham control groups, which may have been due to very low expression levels. The results were consistent with the findings of previous studies, including the study by Stephen et al (14). It is widely accepted that $\mathrm{HCN} 3$ is expressed primarily in the central nervous system and not in the heart; however, the present study did not include the detection of HCN3. Expression of HCN2 and HCN4 was observed in the atria of the sham control and AF groups. Therefore, the results indicate that the majority of $\mathrm{HCN}$ channels in canine atrial muscle are HCN4 and HCN2 isoforms.

Cardiac sinus node pacemaker HCN channels are proteins with important molecular functions (7). Under normal conditions, the expression of $\mathrm{HCN}$ channels in pacemaker cells and the myocardium is relatively low, and the $I_{f}$ current is weak. A variety of pathological factors may stimulate abnormal myocardial expression of HCN channels or cause the development of structural abnormalities. These changes may result in an increase or decrease of the $\mathrm{I}_{\mathrm{f}}$ current, which can in turn cause a variety of arrhythmias. Previous studies have found that mutations in the HCN4 channels of pacemaker cells may cause the $\mathrm{I}_{\mathrm{f}}$ current to weaken and pacemaker cells to self-regulate their heart rate (8-11). In addition, a previous study demonstrated that expression of $\mathrm{HCN} 4$ and $\mathrm{HCN} 2$ channels in ventricular myocytes increases the ventricular $\mathrm{I}_{\mathrm{f}}$ current density, which increases the risk of ventricular arrhythmias (6). $\mathrm{I}_{\mathrm{f}}$ current and $\mathrm{HCN}$ channels have been intensively researched; however, the association between HCN channels and atrial arrhythmias

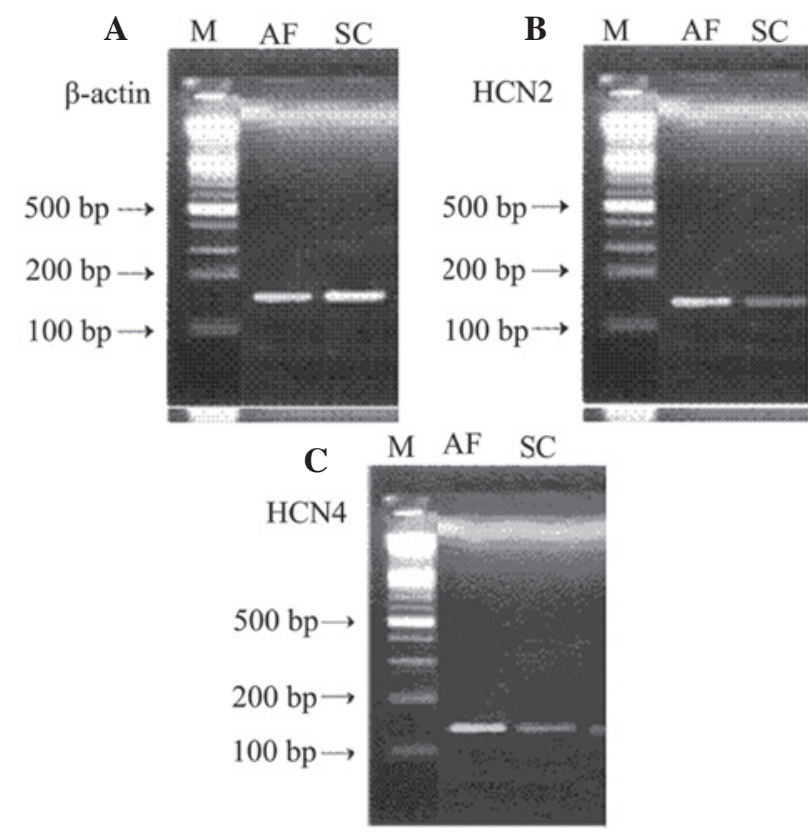

Figure 1. mRNA electrophoresis analysis of (A) $\beta$-actin, (B) HCN2 and (C) HCN4 isoforms. M, marker; AF, atrial fibrillation; SC, sham control; $\mathrm{HCN}$, hyperpolarization-activated cyclic nucleotide-gated.

A

B

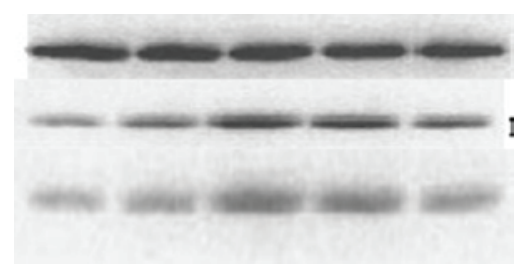

Figure 2. Western blot analysis was used to detect the expression levels of (A) $\beta$-actin (42 kDa), (B) HCN2 (97 kDa) and (C) HCN4 (110 kDa). HCN, hyperpolarization-activated cyclic nucleotide-gated.

remains unknown. Further studies into this association are necessary in order to reveal the role played by HCN channels in cases of AF electrical remodeling. Zorn-Pauly et al (12) found that increases in the $\mathrm{I}_{\mathrm{f}}$ current may cause atrial myocytes to function in a similar manner to pacemaker cells, subsequently increasing the local atrial automaticity, decreasing 
the effective atrial refractory period and enhancing the risk of atrial arrhythmias. The present study found that the mRNA and protein expression levels of $\mathrm{HCN} 2$ and $\mathrm{HCN} 4$ in the AF group increased significantly when compared with the sham control group. Increased expression of these HCN channels in dogs with multiple organ failure and AF can increase the $I_{f}$ current, which may increase atrial automaticity, decrease the effective atrial refractory period and enhance the risk of atrial arrhythmias, with the possibility of causing atrial electrical remodeling (13).

Robinson et al (7) found that increased atrial pacing may lead to an ectopic pacemaker, which is able to progress into a variety of heart conditions, including AF and potentially fatal ventricular arrhythmias. Multiple organ failure can cause the downregulation of a variety of potassium channels, which may be due to the abnormal expression and function of gap junctions, which produces spatial heterogeneity. It was hypothesized that multiple organ failure in dogs with AF, and the subsequent upregulation of HCN2 and HCN4 expression, may result in an ectopic pacemaker, which may in turn progress into a clinically significant arrhythmia. This abnormal pacing may cause cardiac dysfunction due to cellular pathologies, including heart failure, renal failure and multiple organ failure, and is affected by the expression of $\mathrm{HCN}$ channel proteins.

Future studies should focus on multiple organ failure in dogs and examine the range of pathological factors affecting HCN channel expression.

\section{References}

1. Wijffels MC, Kirchhof CJ, Dorland R and Allessie MA: Atrial fibrillation begets atrial fibrillation. A study in awake chronically instrumented goats. Circulation 92: 1954-1968, 1995.
2. Ma CS, Liu XP and Wang Y: Originating from the pulmonary veins electrophysiological characteristics of focal atrial fibrillation with radio frequency ablation. Zhonghua Xin Lu Shi Chang Xue Za Zhi 4: 18-22, 2000 (In Chinese).

3. Gaborit N, Steenman M, Lamirault G, et al: Human atrial ion channel and transporter subunit gene-expression remodeling associated with valvular heart disease and atrial fibrillation. Circulation 112: 471-481, 2005.

4. Baruscotti M, Bucchi A and Difrancesco D: Physiology and pharmacology of the cardiac pacemaker ('funny') current. Pharmacol Ther 107: 59-79, 2005

5. Morillo CA, Klein GJ, Jones DL and Guiraudon CM: Chronic rapid atrial pacing. Structural, functional, and electrophysiological characteristics of a new model of sustained atrial fibrillation. Circulation 91: 1588-1595, 1995.

6. Herrmann S, Stieber J and Ludwig A: Pathophysiology of HCN channels. Pflugers Arch 454: 517-522, 2007.

7. Robinson RB and Siegelbaum SA: Hyperpolarization-activated cation currents: from molecules to physiological function. Annu Rev Physiol 65: 453-480, 2003.

8. Schulze-Bahr E, Neu A, Friederich P, et al: Pacemaker channel dysfunction in a patient with sinus node disease. J Clin Invest 111: $1537-1545,2003$.

9. Ueda K, Nakamura K,Hayashi T, et al: Functional characterization of a trafficking-defective HCN4 mutation, D553N, associated with cardiac arrhythmia. J Biol Chem 279: 27194-27198, 2004.

10. Milanesi R, Baruscotti M, Gnecchi-Ruscone $T$ and DiFrancesco D: Familial sinus bradycardia associated with a mutation in the cardiac pacemaker channel. N Engl J Med 354: 151-157, 2006.

11. Nof E, Luria D, Brass D, et al: Point mutation in the HCN4 cardiac ion channel pore affecting synthesis, trafficking, and functional expression is associated with familial asymptomatic sinus bradycardia. Circulation 116: 463-470, 2007.

12. Zorn-Pauly K, Schaffer P, Pelzmann B, et al: If in left human atrium: a potential contributor to atrial ectopy. Cardiovasc Res 64: 250-259, 2004.

13. Stillitano F, Lonardo G, Zicha S, et al: Molecular basis of funny current (If) in normal and failing human heart. J Mol Cell Cardiol 45: 289-299, 2008.

14. Stephen J, Emerson B, Fox KA and Dransfield I: The uncoupling of monocyte-platelet interactions from the induction of proinflammatory signaling in monocytes. J Immunol 191: 5677-5683, 2013. 